\title{
Modelo de trocas sociais entre tríades de agentes utilizando confiança, reputação e dependência social
}

Social exchange model in triads of agents using trust, reputation and social dependence

\author{
Y. E. León Rojas ${ }^{1}$; G. P. Dimuro ${ }^{1}$; D. F. Adamatti ${ }^{1}$ \\ ${ }^{1}$ Programa de Pós-Graduação em Engenharia de Computação - Centro de Ciências Computacionais, \\ Universidade Federal do Rio Grande (FURG), 96.201-900, Rio Grande-RS, Brazil
}

\{yuniekita, gracaliz,dianaada\}@gmail.com

(Recebido em 19 de setembro de 2014; aceito em 16 de outubro de 2014)

\begin{abstract}
Esse artigo propõe um cenário em que os agentes interagem realizando trocas de serviços que, após serem realizadas, são avaliadas pelos participantes, gerando os conceitos de valores dessas trocas. A teoria desenvolvida por Piaget propõe um modelo de troca social entre dois agentes. Nesse trabalho, se propõe a adição de um novo agente, denominado agente intermediário, que em uma mesma troca atua como fornecedor e recebedor de um serviço. Dessa forma, busca-se complementar o modelo de Piaget, em que as trocas em relações sociais são realizadas entre dois agentes.

Para manter a continuidade do processo de trocas sociais é necessário garantir a existência de trocas futuras e a correta escolha do parceiro. Para tal, precisa-se conhecer a informação dos valores de trocas ocorridas durante o processo, a informação dos agentes com respeito à reputação de cada um dos participantes e o grau de dependência social entre eles. Esses critérios são utilizados para orientar a decisão dos agentes sobre seus parceiros, se convertendo no foco para as análises do processo de transferência de confiança entre os agentes. Pela mesma razão, o conhecimento do grau de confiança e reputação envolvido no processo das trocas promove relações de dependência mais seguras entre os agentes e credibilidade a eles, favorecendo a possibilidade de futuras interações.
\end{abstract}

Palavras-chave: Trocas sociais, Sistema multiagentes, Reputação, Confiança.

This article proposes a scene where the agents interact among them to exchange services that, after being done, are evaluated by the participants creating the concept of value of the exchanges. The theory developed by Piaget proposes a social exchange model between two agents. This work proposes the addition of a new one, called intermediary agent, which in the same exchange acts as provider and receiver of a service. This way, the basic model proposed by Piaget, where the social exchanges are made among three agents, can be complemented.

To keep the social exchanges process continuity, it is necessary to ensure the future exchanges existence and the correct partner choice. For this, it is necessary to get the information of the values happening during the process, the information of the agents related to the reputation of each of the participants and the social dependency level among them. These criteria are used to orient the agents decision over their partners, getting a target to the confidence transfer process analysis among the agents. For the same reason, the confidence and reputation level knowledge involved in the exchanges process promotes more secure dependency relationships among the agents and credibility, making future interactions possible.

Keywords: Multiagents Systems, Social Exchanges, Trust.

\section{INTRODUÇÃO}

Trocas sociais em sistemas multiagentes podem ser entendidas como processos de interações entre pares de agentes, por meio de trocas de serviços. A troca consiste na realização ou pagamento de um serviço de um agente para outro, esse processo gera em cada iteração valores de trocas, investimento, satisfação, custo e beneficio, como resultado da avaliação do serviço prestado [1]. Assim, os valores gerados permitem que um agente tome uma atitude frente aos demais para a escolha dos parceiros em suas trocas futuras [2][3][4][5].

Dentro dos sistemas multiagentes, as trocas sociais têm sido utilizadas como base para análises de interações dos agentes [6][7][8][9]. Também foram observados diferentes trabalhos referentes às interações sociais que ressaltam que os agentes são entidades que encontram-se em ambientes físicos ou simulados, percebendo seu ambiente, com autonomia de decisão e de 
execução, além disso, cada agente encontra-se formando coalizões, delegando tarefas, cooperando e coordenando com outros agentes [10].

Diversos conceitos são relacionados a esses processos de troca, tais como as relações de dependência que podem surgir entre os agentes, a formação da reputação de cada agente envolvido na troca e a confiança. Consequentemente, para a escolha dos parceiros, uma das fontes da informação que o agente vai ter está baseada nas relações dependência que ele tem com os outros agentes. Assim, os estudos mostram que a relação de dependência se dá quando um agente quer alcançar um estado, que é o seu objetivo, mas não tem a possibilidade de alcança-lo. Portanto, ele procura um segundo agente que tenha todas as condições necessárias para ajudá-lo a alcançar esse estado [11]. E, além das informações sobre as relações de dependência, existe a informação gerada pela reputação de cada agente envolvido na troca, a qual pode ser qualificada como se mostra em [12] e [13]. Essa informação é necessária para a escolha do parceiro. A transferência de informação entre os agentes influencia na confiança e na reputação, e vice-versa. Isto ocorre porque a reputação é um fator envolvido na construção da confiança e a confiança apresenta-se como uma relação entre agentes que tem como parte de seu estado mental crenças e objetivos [14] [15] [16] [17] [18].

Obervando-se os estudos realizados, logo se nota que os Sistemas multiagentes têm a capacidade de desempenhar um papel importante no desenvolvimento e análise de modelos e teorias de interatividade nas sociedades humanas. Os seres humanos interagem de várias maneiras e em muitos níveis, por exemplo, solicitando e fornecendo informações, negociando e discutindo, desenvolvendo visões compartilhadas de um ambiente, formando ou dissolvendo estruturas organizacionais.

Para manter a continuidade do processo de trocas sociais é necessário garantir a existência de trocas futuras e a correta escolha do parceiro. Para tal, precisa-se conhecer a informação dos valores de trocas ocorridas durante o processo, a informação dos agentes com respeito à reputação de cada um dos participantes e o grau de dependência social entre eles.

Essa proposta integra sistemas multiagentes, trocas sociais, dependência, reputação e confiança, não tratando esses fatores isoladamente. Dessa forma, pode-se desenvolver um modelo de trocas sociais em tríades de agentes para avaliar o processo de transferência de confiança baseado nos conceitos de reputação e relações de dependência em sistemas multiagentes, complementando assim o modelo básico das trocas socais de Piaget com adição de um terceiro agente para análise de aspectos não econômicos do processo da troca.

\section{REFERENCIAL TEÓRICO}

Este artigo se fundamenta nos conceitos de trocas sociais, confiança, dependência e reputação dentro dos sistemas multiagentes, com o fim de modelar as relações de dependência e reputação entre agentes e levando a analise a possibilidade da transferência da confiança em tríades de agentes.

\subsection{SISTEMAS MULTIAGENTES}

Os sistemas multiagentes, utilizados geralmente para modelar e simular sistemas complexos, estão formados por agentes autônomos que, por um lado têm a capacidade de interagir entre eles e com o ambiente onde se encontram, e por outro, tem a capacidade de observar e comunicar-se atuando de modo cooperativo a fim de atingir metas ou objetivos. Ou seja, cada agente tem existência própria independente dos outros agentes.

No contexto dos agentes cognitivos, existem os agentes BDI, que são agentes que têm a capacidade de realizar ações independentes e autônomas, decidem por si o que fazer e atendem determinados objetivos para os quais foram projetados. O modelo de agentes BDI (belief desire - intention) é representado por uma arquitetura cognitiva baseada em estados mentais: crenças, desejos e intenções, que derivam de processos chamados raciocínios práticos. Crenças (beliefs) são as informações que o agente possui a respeito do ambiente que o rodeia, representando seu conhecimento. Um agente pode ter crenças sobre outros agentes, sobre interações com outros agentes e crenças sobre suas próprias crenças. Desejos (desires) são 
estados motivacionais do agente, representando os objetivos ou metas que o agente gostaria de realizar. As intenções (Intentions) representam sequências de ações específicas ou planos que um agente pode escolher para atingir um determinado objetivo [19]. Ou seja, uma vez formado o objetivo (desejo), o agente tenta realizá-lo por meio de um plano (intenção), levando em consideração a informação do meio em que se encontra (crenças) e persistindo até que seu desejo se realize.

\subsection{TROCAS SOCIAIS}

As trocas sociais são compreendidas como trocas de serviços entre pares de agentes, $\mathrm{X}$ e $\mathrm{Y}$, em duas etapas ou estágios de trocas [1]. Na Etapa I o agente $\mathrm{X}$ realiza um serviço para o agente $\mathrm{Y}$ e na Etapa II o agente $\mathrm{X}$ solicita para o agente $\mathrm{Y}$ o pagamento do serviço realizado anteriormente para ele. Gerando-se em cada Etapa valores de trocas, produto da avaliação do serviço realizado. Os valores são compreendidos como construções mentais de natureza qualitativa, que expressam uma situação subjetiva, armazenada na consciência, após de uma situação de troca.

$\mathrm{Na}$ etapa $\mathrm{I}$ o agente $X$ realiza um serviço com algum valor de investimento $\left(\mathrm{R}_{\mathrm{x}}\right)$ para $\mathrm{o}$ agente $\mathrm{Y}$. $\mathrm{O}$ agente $\mathrm{Y}$ gera pelo serviço recebido um valor de satisfação $\left(\mathrm{S}_{\mathrm{y}}\right)$ e um valor de reconhecimento $\left(\mathrm{T}_{\mathrm{y}}\right)$ ou débito pela satisfação com o serviço recebido de $\mathrm{X}$. No final desta etapa o agente $\mathrm{X}$ acaba com um valor virtual $\left(\mathrm{V}_{\mathrm{x}}\right)$, ou seja, crédito devido à ação que realizou em favor do agente $Y$. Na etapa II existe uma possível cobrança de dívida do agente $\mathrm{X}$ ao agente $\mathrm{Y}$, onde $\mathrm{o}$ agente $\mathrm{X}$ cobra do agente $\mathrm{Y}$ um serviço relativo ao seu valor virtual $\left(\mathrm{V}_{\mathrm{x}}\right)$ de crédito adquirido na Etapa I. $\mathrm{O}$ agente $\mathrm{Y}$ por sua vez possui em sua consciência um valor de débito $\left(\mathrm{T}_{\mathrm{y}}\right)$, realizando uma oferta com valor de investimento $\left(R_{y}\right)$ para o agente $X$, que gerará um valor de satisfação $\left(S_{\mathrm{x}}\right)$ referente à oferta de $\mathrm{Y}$ (ver a Figura 1$)$.

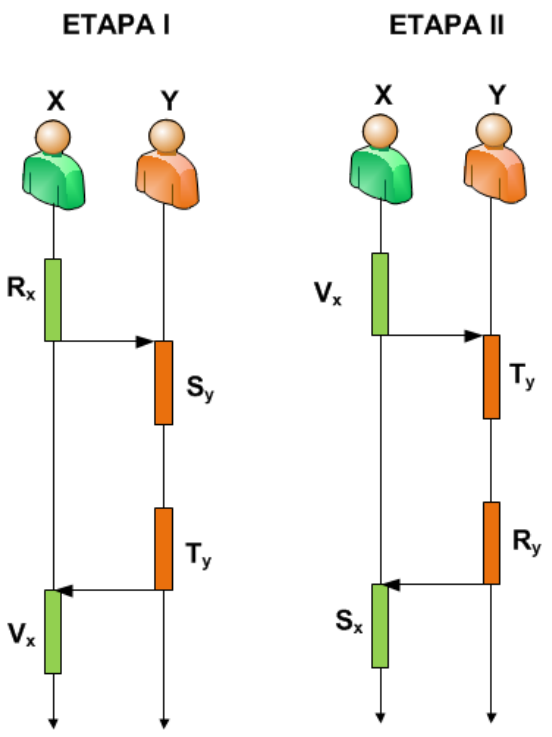

Figura 1: Etapas I e II das trocas sociais de Piaget entre dois agentes.

\subsection{CONFIANÇA E REPUTAÇÃO}

O termo confiança possui definições de distintos autores, dependendo de cada área de estudo (comércio, política, tecnologia, organização, segurança, etc.), sem existir ainda uma noção compartilhada ou predominante. Entre elas, a confiança pode ser definida como a expectativa que uma pessoa tem sobre a ação das outras pessoas [20]. Também é definida como uma atitude, sendo naturalmente fundamentada nas crenças relativas às características únicas da outra parte e de elementos de uma situação específica [21]. Segundo este autor, as crenças no parceiro são derivadas de experiências anteriores e as consequências da confiança são traduzidas em um determinado comportamento ou intenção de ação. Em suma, a confiança é considerada 
como uma atitude e uma relação social entre os agentes envolvidos, que leva em consideração a decisão e a delegação, definindo-a como um estado mental que apresenta ingredientes mentais, tais como crenças e objetivos, que compõe um agente BDI [22].

Tal qual a confiança, o termo reputação também possui vários conceitos, nas mais diversas áreas do conhecimento. Para as ciências sociais, as reputações são definidas como um coletivo de crenças e opiniões que influenciam as ações dos indivíduos em relação aos seus pares. A reputação pode ainda ser vista como uma ferramenta social com o objetivo de reduzir a incerteza de se interagir com indivíduos de atributos desconhecidos. A reputação é um dos três pilares da psicologia social. Os outros dois são a personalidade e o relacionamento entre as pessoas e grupos [23]. Pode-se dizer, que a reputação é também definida como a quantidade de confiança inspirada por uma determinada pessoa em um ambiente ou domínio específico de interesse [24].

Em suma, pode-se dizer que a confiança é a expectativa que um agente tem sobre a ação dos outros agentes, e a reputação consiste na transmissão de crenças sobre como os agentes são avaliados em relação a uma conduta desejada.

Modelos de confiança e reputação, dentro da ciência da computação, têm ganhado crescente evidência nos últimos anos, principalmente no ramo da Inteligência Artificial Distribuída (IAD). A confiança e reputação são utilizados como um meio de busca de parceiros. A reputação tem o poder de propagar a confiança e pode evitar que os agentes interajam desnecessariamente.

A maioria dos modelos sobre reputação tem uma representação quantitativa, onde a reputação é um valor, que pode ignorar aspectos da sua construção, importantes para os agentes, que poderiam fornecer um estado de confiança mais completo sobre determinado agente. Também existem modelos que promovem uma análise qualitativa da reputação, baseada nos componentes que a compõem, permitindo ao agente chegar a um estado interno de confiança.

Muitos modelos computacionais e teóricos, e abordagens de confiança e reputação vêm sendo desenvolvidos nos últimos anos. A Tabela 1 apresenta alguns desses modelos em SMA.

Tabela 1: Quadro comparativo dos modelos para confiança e reputação.

\begin{tabular}{|c|c|c|c|}
\hline \multirow[b]{2}{*}{ Modelo } & \multicolumn{3}{|c|}{ Classificação } \\
\hline & Origem da informação & Visibilidade & Tipo de modelo \\
\hline SPORAS & Testemunhos & Global & Reputação \\
\hline Hitos & Direta + Testemunhos & Individual & Reputação \\
\hline ReGret & Direta + Testemunhos + & Individual & Reputação e Confiança \\
\hline Marsh & Direta & Individual & Confiança \\
\hline Travos & Direta + Testemunhos & Individual & Reputação e Confiança \\
\hline Afras & Direta + Testemunhos & Individual & Reputação \\
\hline $\begin{array}{l}\text { Castelfranchi e } \\
\text { Falcone }\end{array}$ & Não especifica origem & Individual & Confiança \\
\hline ForTrust & Não especifica origem & Individual & Reputação e confiança \\
\hline
\end{tabular}

\subsection{DEPENDÊNCIA}

As iterações sociais são caracterizadas por ser imprevisíveis e ilimitadas. Neste sentido, a teoria da dependência de relações pode ser utilizada como um critério de decisão para a escolha de um parceiro em um SMA [25]. Esta teoria permite controlar as iterações sociais, onde cada iteração é denominada como dependência social, que envolve relações de dependência. Um dos aspectos mais importantes das relações dependência é que possibilita prever outras relações sociais [26]. Segundo a teoria das dependências, um agente $X$ deve raciocinar sobre a maneira correta de delegar uma ação que necessita para outro agente, garantindo que essa ação seja realizada e que o agente que recebe essa ação esteja consciente que está realizando a mesma para que o agente alcance seu objetivo. 


\section{MODELO PROPOSTO}

O modelo de troca proposto, inspirado na Teoria de Piaget é formado por duas etapas (I e II) e composto por 3 agentes, X, D e Y, denominado de Troca Social com Agente Intermediário (TSAI).

$\mathrm{Na}$ Etapa I ocorrem dois processos de troca, separados em Troca 1 e Troca 2, enquanto na Etapa II existe um processo de troca dividida em duas partes.

$\mathrm{Na}$ Etapa I, Figura 3, o agente D, denominado como agente intermediário, gera valores materiais: de investimento $(\mathrm{R})$ e de satisfação $(\mathrm{S})$, e valores virtuais: de crédito $(\mathrm{V})$ e de débito(T), consequência das trocas imediatas e de trocas futuras que virá a participar. Assim, ele participa de duas trocas (Troca 1 e Troca 2), comportando-se como um agente que recebe um serviço em uma delas e como um agente que outorga um serviço na outra.

$\mathrm{O}$ agente $\mathrm{X}$, na Troca 01, vai possuir valores materiais (de investimento, $\mathrm{R}_{\mathrm{xd}}$ ) e valores virtuais (de crédito, $\mathrm{V}_{\mathrm{xd}}$ ).

$\mathrm{O}$ agente $\mathrm{Y}$, na Troca 02 , possui valores materiais (de satisfação, $\mathrm{S}_{\mathrm{yd}}$ ) e valores virtuais (de débito, $\left.\mathrm{T}_{\mathrm{yd}}\right)$.

\section{Etapa I - Troca 01:}

1. $R_{x d}$ : Valor do investimento do agente $X$ na realização de um serviço para agente $D$.

2. $\mathrm{S}_{\mathrm{dx}}$ : Valor de satisfação do agente $\mathrm{D}$ pelo serviço recebido do agente $X$.

3. $\mathrm{T}_{\mathrm{dx}}$ : Valor do débito do agente $\mathrm{D}$ pela satisfação do serviço recebido por $\mathrm{X}$.

4. $\mathrm{V}_{\mathrm{xd}}$ : Valor do crédito que $\mathrm{o}$ agente $\mathrm{X}$ adquiriu com $\mathrm{D}$ pela realização de um serviço.

Etapa I - Troca 02:

1. $R_{\mathrm{dy}}$ : Valor do investimento do agente $\mathrm{D}$ na realização de um serviço para agente $Y$.

2. $S_{y d}$ : Valor de satisfação do agente $Y$ pelo serviço recebido do agente $D$.

3. $\mathrm{T}_{\mathrm{yd}}:$ Valor do débito do agente $\mathrm{Y}$ pela satisfação do serviço recebido por $\mathrm{D}$.

4. $V_{\text {dy }}$ : Valor do crédito que o agente $\mathrm{D}$ adquiriu com $\mathrm{Y}$ pela realização de um serviço.

ETAPA I

Troca 1

Troca 2
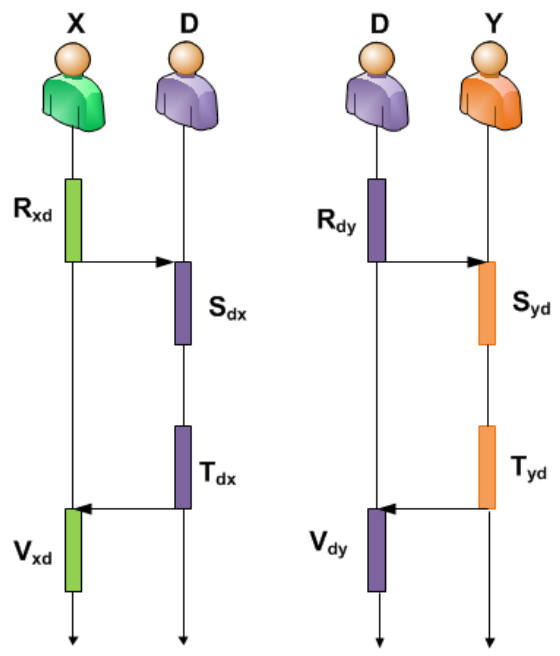

Figura 3: Etapa I de trocas entre três agentes.

$\mathrm{Na}$ Etapa II, Figura 4, o agente intermediário D possui um valor virtual (de débito, $\mathrm{T}_{\mathrm{dx}}$ ) e um valor material (de investimento, $R_{\mathrm{dx}}$ ). $\mathrm{O}$ valor $\mathrm{R}_{\mathrm{dx}}$ vai estar em função do valor de débito $\left(\mathrm{T}_{\mathrm{dx}}\right)$ pelo serviço recebido de $\mathrm{X}$ e um valor de crédito $\left(\mathrm{V}_{\mathrm{dy}}\right)$ realizado para $\mathrm{Y}$, dado por $\mathrm{R}_{\mathrm{dx}}=\mathrm{F}_{1}\left(\mathrm{~T}_{\mathrm{dx}} ; \mathrm{V}_{\mathrm{dy}}\right)$. 
$\mathrm{O}$ agente $\mathrm{X}$ vai possuir um valor virtual (de crédito, $\mathrm{V}_{\mathrm{xd}}$ ) e um valor material (de satisfação, $S_{x d}$, gerado ao receber o serviço. Esse valor vai estar em função do investimento feito pelo agente $\mathrm{Y}$ para o agente $\mathrm{D}$, dado por $\mathrm{S}_{\mathrm{xd}}=\mathrm{F}_{2}\left(\mathrm{~S}_{\mathrm{dy}}\right)$.

$\mathrm{O}$ agente $\mathrm{Y}$ possui um valor virtual (de débito, $\mathrm{T}_{\mathrm{yd}}$ ) e um valor material (de investimento, $\left.\mathrm{R}_{\mathrm{yd}}\right)$.

\section{Etapa II}

1. $\mathrm{V}_{\mathrm{xd}}$ : Valor do crédito do agente $\mathrm{X}$ na realização de um serviço para agente $\mathrm{D}$.

2. $\mathrm{T}_{\mathrm{dx}}$ : Valor de débito do agente $\mathrm{D}$ pelo serviço recebido do agente $\mathrm{X}$.

3. $\mathrm{R}_{\mathrm{dx}}$ : Valor do investimento do agente $\mathrm{D}$ para com $\mathrm{X}$ pela satisfação com serviço recebido, em função do valor $\mathrm{T}_{\mathrm{dx}}$ respeito com o serviço de oferecido por $\mathrm{X}$ e do valor $\mathrm{V}_{\mathrm{dy}}$ entregue por D para $\mathrm{Y}$.

4. $\mathrm{T}_{\mathrm{yd}}:$ Valor do débito do agente $\mathrm{Y}$ para com $\mathrm{D}$ pela satisfação com o serviço recebido.

5. $\mathrm{R}_{\mathrm{yd}}$ : Valor de investimento do agente $\mathrm{Y}$ na realização de um serviço para agente $\mathrm{D}$.

6. $S_{\mathrm{dy}}$ : Valor de satisfação do agente $\mathrm{D}$ adquiriu com $\mathrm{Y}$ pela realização de um serviço.

7. $S_{x d}$ : Valor de satisfação do agente $X$ adquiriu com $D$ pela realização de um serviço, em função de investimento de Y para D.

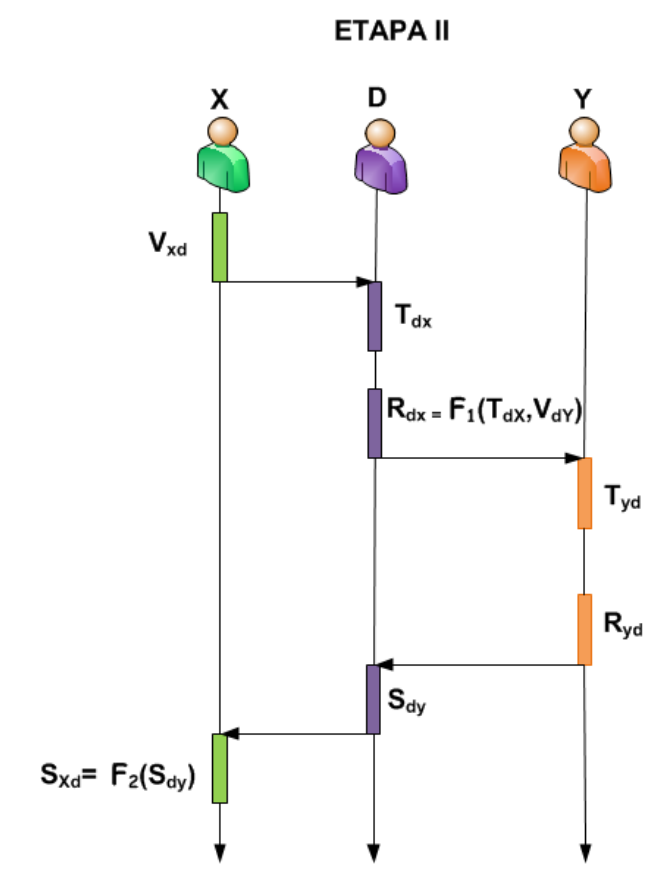

Figura 4: Etapa II de trocas entre três agentes.

Esse tipo de processo de troca, no qual participam os três agentes, será denominado Troca Social com Agentes Intermediários (TSAI) e é caracterizada por um agente intermediário (D) que não tem a possibilidade de fazer o pagamento da dívida que tem com o agente $\mathrm{X}$ e vai procurar um terceiro agente, Y, com que ele teve um processo de troca (Etapa I) para realizar o serviço que ele tem como divida.

É importante enfatizar que não existe uma ordem na ocorrência das Etapas I.

\subsection{RELAÇÕES DE DEPENDÊNCIA EM TSAI}

No ambiente em que os agentes $\mathrm{X}, \mathrm{D}$ e $\mathrm{Y}$ interagem, trocando serviços a fim de alcançar objetivos individuais, o agente $\mathrm{D}$ vai se comportar como um agente intermediário que tem uma lista de serviços de que pode executar e uma lista de serviços que pode oferecer (cliente e provedor ao mesmo tempo).

$\mathrm{O}$ agente $\mathrm{X}$ não precisa se relacionar diretamente com o agente $\mathrm{Y}$, ou seja, quando o agente $\mathrm{X}$ precisar de um serviço, ele tem a possibilidade de escolher outro agente, como o agente $\mathrm{D}$, com 
quem tem histórico de valores de trocas, que demonstram potencial para realizar o serviço e favorecer a possibilidade de trocas futuras. $\mathrm{D}$ pode não ter a possibilidade de realizar a solicitação de $\mathrm{X}$, mas pode terceirizar o serviço para $\mathrm{Y}$, com quem também tem histórico de valores de trocas e relações.

Um processo de tomada de decisão para a seleção de parceiros deve estabelecer não só o processo de escolha de um parceiro, como também o processo de análise das solicitações de serviços recebidas, levando em consideração os valores de trocas. Ou seja, o agente deve escolher agentes para enviar uma solicitação de serviço, baseado em relações de dependência estabelecidas, dando preferência aos agentes mais propensos a aceitar o pedido. A Figura 5 mostra o processo de uma troca de dependência em TSAI.

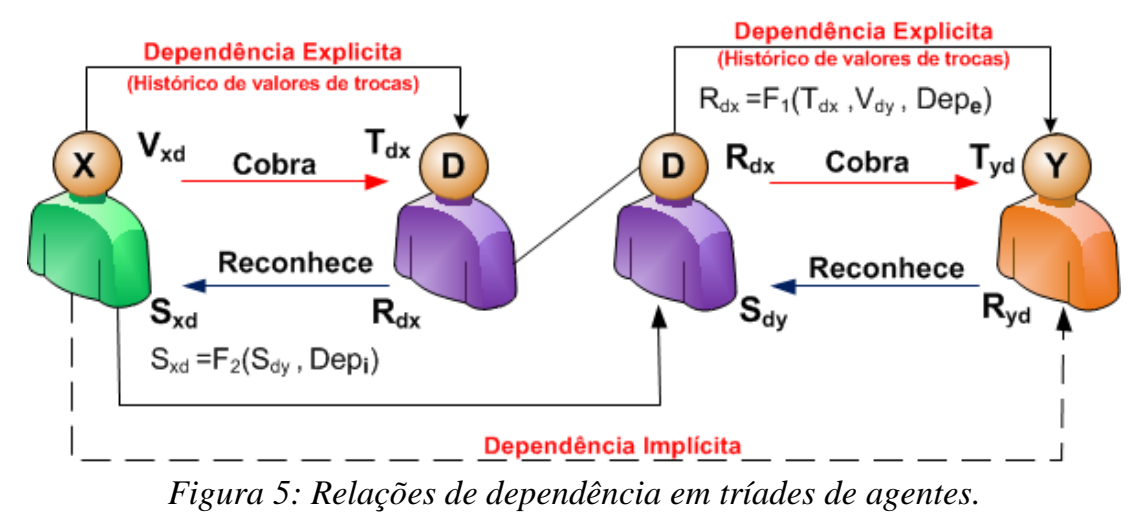

$\mathrm{O}$ agente $\mathrm{X}$, por meio de um processo de troca, investe $\mathrm{R}_{\mathrm{dx}}$ para a realização de um serviço para o agente $\mathrm{D}$, somente se $\mathrm{D}$ for capaz de retornar um serviço que $\mathrm{X}$ precisaria. $\mathrm{O}$ agente $\mathrm{D}$, da mesma maneira que $\mathrm{X}$ realiza um investimento para realizar um serviço para $\mathrm{Y}$. O agente $\mathrm{D}$ por meio de um processo de troca tem um valor de Débito $\mathrm{T}_{\mathrm{dx}}$ com o agente $\mathrm{X}$, então pode-se dizer que o agente D é susceptível a realização de um serviço para o agente X. Impossibilitado de realizar o serviço, o agente D solicita a execução do serviço a um terceiro agente, procurando aquele com o qual ele identifica uma dependência ou que é visto como mais propenso a colaborar. Para tal, são levados em conta os níveis de dependência (Dep) e histórico de valores de trocas $(\mathrm{R}, \mathrm{S}, \mathrm{T}, \mathrm{V})$. Nessa configuração, a relação entre os agentes $\mathrm{D}$ e $\mathrm{Y}$ é chamada de dependência explicita Depe, enquanto a relação dos agentes X e Y é chamada de dependência implícita Dep . $_{\text {. }}$

\subsection{CRENÇAS DE GRUPO SOBRE A REPUTAÇÃO EM TSAI}

Para dar início à construção das crenças de grupo sobre a reputação em tríades de agentes, $X$, $\mathrm{D}$ e $\mathrm{Y}$, durante o processo de trocas sociais, serão analisadas as seguintes relações: o agente $\mathrm{X}$ cobra o agente $\mathrm{D}$ pelo serviço prestado na primeira etapa, e para isto X envia a D o valor de crédito que se acha merecedor. $\mathrm{X}$ analisa $\mathrm{D}$ por meio de uma comparação entre seu valor de crédito e o valor de débito de $\mathrm{D}$, com o fim de saber se é maior, menor ou igual ao valor de débito que atribuiu a si mesmo. A análise de $\mathrm{X}$ é feita sob os valores do processo de trocas geradas com D na primeira etapa. Consecutivamente, o agente $\mathrm{D}$ também faz uma análise do valor de crédito que possui com o agente $\mathrm{Y}$, da mesma maneira feita por $\mathrm{X}$, com o agente $\mathrm{D}$, no instante em que é realizada a cobrança do serviço.

Finalizada a análise de cobrança e considerando os valores de crédito e débito, o agente $\mathrm{X}$ envia informação sobre o agente $\mathrm{D}$ e o agente $\mathrm{D}$ envia informação sob o agente $\mathrm{Y}$ para $\mathrm{o}$ registro de crenças de grupo, para assim formar uma reputação sobre os dois agentes.

Por fim, quando o agente $\mathrm{Y}$ reconhece o serviço e gera um valor de satisfação para o agente $\mathrm{D}$ e indiretamente para o agente $\mathrm{X}$, o agente $\mathrm{D}$ envia uma informação sobre o agente $\mathrm{Y}$ para atualizar o registro de crenças de grupo e para atualizar a reputação sobre Y. Isso é feito após a análise de comparação entre os valores de crédito com Y e o valor de satisfação gerada por Y. 
Da mesma forma, o agente $\mathrm{X}$ realiza análises sobre o agente $\mathrm{D}$, levando em conta o valor de crédito e o valor de satisfação recebido. Neste contexto, o agente $X$ cria uma reputação direta $\left(\operatorname{Rep}_{\mathrm{d}}\right)$ a respeito do agente $\mathrm{D}$ e uma reputação indireta $\left(\operatorname{Rep}_{\mathrm{i}}\right)$ a respeito do agente $\mathrm{Y}$, e o agente $\mathrm{D}$ creia uma reputação direta a respeito do agente Y. Todos esses dados são enviados como informação ao registro de crenças de grupo, atualizando a reputação de cada um dos agentes, como se mostra na Figura 6

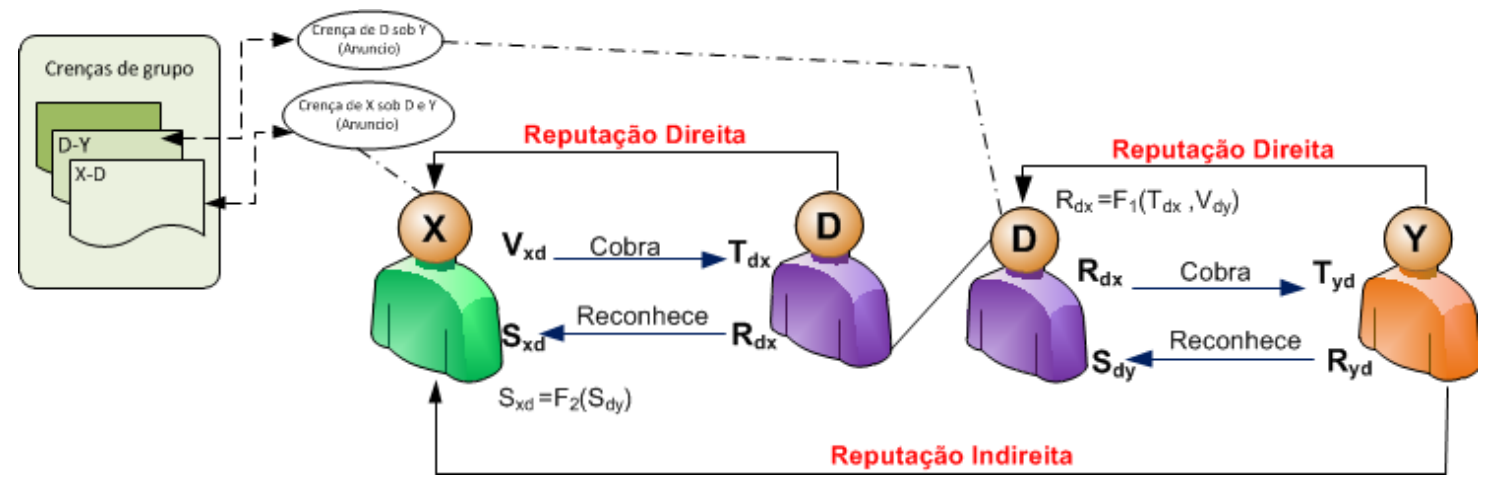

Figura 6: Relações de Reputação em tríades de agentes.

1) $X$ cobra de $D$ o serviço prestado na Etapa $I$ - Troca 1 .

$\mathrm{X}$ envia para $\mathrm{D}$ o valor que se acha merecedor $\left(\mathrm{V}_{\mathrm{xd}}\right)$.

$\mathrm{X}$ analisa $\mathrm{D}$ por comparação $\mathrm{V}_{\mathrm{xd}}=,<,>\mathrm{T}_{\mathrm{dx}}$

Registro de crenças (X,D).

2) D cobra de $Y$ o serviço prestado na Etapa I - Troca 2.

$\mathrm{D}$ envia para $\mathrm{Y}$ o valor que se acha merecedor $\left(\mathrm{V}_{\mathrm{dy}}\right)$.

D analisa $\mathrm{Y}$ por comparação $\mathrm{V}_{\mathrm{dy}}=,<,>\mathrm{T}_{\mathrm{yd}}$

Registro de crenças (D,Y).

3) $Y$ gera para $D$ o valor de satisfação $S_{d y}$ e indiretamente para $X$ o valor $S_{x d}$

$\mathrm{D}$ analisa $\mathrm{Y}$ por comparação $\mathrm{V}_{\mathrm{dy}}=,<,>\mathrm{S}_{\mathrm{dy}}$

$\mathrm{X}$ analisa $\mathrm{D}$ por comparação $\mathrm{V}_{\mathrm{xd}}=,<,>\mathrm{S}_{\mathrm{dx}}$

$\mathrm{X}$ e $\mathrm{D}$ atualizam o registro de crenças.

\subsection{TRANSFERÊNCIA DE CONFIANÇA EM TSAI}

O modelo será baseado em crenças individuais constituídas por objetivos, capacidades, competências e intenções de cada agente. $\mathrm{O}$ agente intermediário, que não tem a possibilidade de realizar o serviço, deverá estar disposto a confiar em um terceiro agente para executar uma determinada ação. Conforme o modelo mostrado na Figura 3, o agente X está disposto a confiar no agente $\mathrm{D}$, se e somente se, $\mathrm{X}$ pensa que possivelmente irá precisar de uma ação de $\mathrm{D}$ no futuro para alcançar um determinado objetivo sob alguma circunstancia. No processo de trocas sociais, essa situação vai estar representada por valores de débito (T). Assim, no processo chamado de Etapa II das trocas sociais, mostrado na Figura 4, observa-se:

\section{Agente $X$ com respeito ao Agente D:}

1. $X$ tem um objetivo potencial $\left(\mathrm{V}_{\mathrm{dx}}\right)$ para alcançar em determinadas circunstâncias $\left(\mathrm{T}_{\mathrm{dx}}\right)$;

2. X confia que, se quiser alcançar o objetivo e as circunstâncias não mudam, então:

a. D será capaz de fazer uma ação $\mathrm{R}_{\mathrm{dx}}$

b. $\mathrm{D}$, realizando a ação $\mathrm{R}_{\mathrm{dx}}$ assegura alcançar o objetivo $\mathrm{V}_{\mathrm{xd}}$, obtendo um valor de satisfação $S_{x d}$ desejado por X.

c. $\mathrm{D}$ Tem a intenção de fazer $\mathrm{R}_{\mathrm{dx}}$ porque tem uma dívida com $\mathrm{X}$.

\section{Agente D com respeito ao Agente Y:}

Como D não pode realizar o serviço solicitado por X, ele busca um terceiro agente $\mathrm{Y}$ que possa realizá-lo. O mesmo que ocorreu entre $\mathrm{X}$ e $\mathrm{D}$, volta a ocorrer entre $\mathrm{D}$ e $\mathrm{Y}$.

1. $\mathrm{D}$ tem um objetivo potencial $\left(\mathrm{V}_{\mathrm{dy}}\right)$ para alcançar em determinada circunstância $\left(\mathrm{T}_{\mathrm{yd}}\right)$; 
2. D confia que, se quiser alcançar o objetivo de pagar a dívida com $X$ e as circunstâncias não mudam, então:

a. Y será capaz de fazer una ação $R_{\mathrm{yd}}$.

b. $Y$ realizando a ação $R_{y d}$, assegura alcançar o objetivo de $X, V_{x d}$, obtendo um valor de satisfação $S_{d x}$ desejado por $X$ e $S_{d y}$ desejado por $D$.

c. $\mathrm{Y}$ tem uma predisposição a fazer $\mathrm{R}_{\mathrm{dy}}$ porque tem uma dívida com $\mathrm{D}$.

\section{Agente $\mathrm{X}$ com respeito ao Agente $\mathrm{Y}$ :}

Como, pela transferência da confiança, o agente $\mathrm{X}$ vai acreditar em $\mathrm{Y}$ após obter o pagamento do crédito solicitado para o agente $\mathrm{D}$ e concretizado pelo agente $\mathrm{Y}$, pode-se dizer que:

1. $X$, se tiver um objetivo potencial $(V)$ para alcançar em determinadas circunstâncias $(T)$, acredita que, se quiser alcançar o objetivo e as circunstâncias não mudam, então:

a. Y será capaz de fazer uma ação R.

b. Y, realizando a ação R assegura alcançar o objetivo (V) de X, e conseguirá obter o valor de satisfação S desejado.

c. Y tem a intenção de fazer um investimento, R, como parte de um processo de troca social.

\section{CONCLUSÃO}

No mundo real é muito comum a existência de intermediários (terceiro agente) no processo de trocas. É importante considerar a extensão da teoria das Trocas Sociais de Piaget para três agentes e analisar o processo da transferência de confiança entre os agentes envolvidos.

Os modelos apresentados são o início para a análise dos aspectos não econômicos dos processos de trocas sociais, pois analisa as informações dos valores de trocas gerados pela avaliação do serviço feita pelos agentes e como as trocas sociais vão ser influenciadas pela reputação e relações de dependência existentes.

No estágio atual da pesquisa, a análise é feita por meio de uma formalização teórica (modelos). Posteriormente, deseja-se realizar simulações e avaliações por meio de um estudo de caso, de forma a validar quantitativamente o modelo proposto.

\section{REFERÊNCIA BIBLIOGRÁFICAS}

1. Piaget J. Sociological Studies London: Routlege; 1995.

2. Dimuro GP, Costa AC, Palazzo L. Systems of Exchange Values as Tools for Multi-Agent Organizations. Journal of the Brazilian Computer Society. 2005 July; 27-40.

3. Macedo LF, Dimuro GP, Aguiar MS, Costa AC, Mattos VL, Coelho H. Game, Analyzing the Evolution of Social Exchange Strategies in Social Preference-Based MAS through an Evolutionary Spatial Approach of the Ultimatum. Third Brazilian Workshop on Social Simulation. 2012 October; 83-90.

4. Rodrigues MR, Luck M. Analysing Partner Selection Through Exchange Values. Multi-Agent-Based Simulation VI. 2006 July; 24-40.

5. Costa AR. A teoria piagetiana das trocas sociais e sua aplicação aos ambientes de ensinoaprendizagem. Informática na educação: teoria \& prática. 2003 Julio; 77-90.

6. Farias GP, Dimuro GP, Dimuro G, De Manuel Jerez E. Exchanges of Services based on Piaget's Theory of Social Exchanges using a BDI-Fuzzy Agent Model. Computational Intelligence and 11th Brazilian Congress on Computational Intelligence (BRICS-CCI \& CBIC). 2013 September; 653-658.

7. Dimuro GP, Costa AC, Palazzo L. Systems of exchange values as tools for multi-agent organizations. Journal of the Brazilian Computer Society. 2005 July; 27-40.

8. Dimuro GP, Costa AR, Gonçalves L, Pereira D. Recognizing and learning models of social exchange strategies for the regulation of social interactions in open agent societies. Journal of the Brazilian Computer Society. 2011 January; 143-161.

9. Rodrigues MR, Luck M. Effective Multiagent Interactions for Open Cooperative Systems Rich in Services. In 8th International Joint Conference on Autonomous Agents and Multiagent Systems (AAMAS 2009); 2009; Budapest. p. 1273--1274. 
10. Pereira D, Gonçalves L, Dimuro G, Costa A. Towards the Self-regulation of Personality-Based Social Exchange Processes in Multiagent Systems. Advances in Artificial Intelligence - SBIA 2008. 2008 October: p. 113-123.

11. Sichman JS, Demazeau Y. first attempt to use dependence situatons as a decision criterion for choosing partners in multi-agent systems. In 94 WorkShop on Decision Theory for Dai Aplications; 1994.

12. Schmitz TL. Crenças de grupo como instrumento de formação da reputação: uma arquitetura baseada em agentes e artefatos. Dissertação de Mestrado. Florianópolis: Universidade Federal de Santa Catarina, Santa Catarina; 2011.

13. Von Laer A. Autorregulação de processos de trocas sociais em SMA: um modelo de sociedade de agentes BDI evolucionários e culturais no contexto de sociedade de agentes BDI evolucionários e culturais no contexto do JaCaMo. Dissertação de Mestrado. Rio Grande: Universidade Federal do Rio Grande, Rio Grande do Sul; 2014.

14. Castelfranchi C, Falcone R, Firozabadi B, Tan Y. Special issue on trust, deception and fraud in agent societies. Applied Artificial Intelligence Journal. 2000; 763-768.

15. Falcone R, Castelfranchi C. Principles of trust for MAS: Cognitive anatomy, social importance and quantification. International Conference of Multi-agent Systems (ICMAS). 1998 July; 72-79.

16. Falcone R, Castelfranchi C. Social Trust: A Cognitive Approach. Trust and Deception in Virtual Societies. 2001; 55-90.

17. Al-Mutairi MS, Hipel KW, Kamel MS. Trust and cooperation from a fuzzy perspective. Mathematics and Computers in Simulation. 2008 April; 430-446.

18. Martínez J, Pavón J. Modelling trust into an agent-based simulation tool to support the formation and configuration of work teams. 7th International Conference on Practical Applications of Agents and Multi-Agent Systems (PAAMS 2009). 2009; 80-89.

19. Wooldridge M. Reasoning about rational agents: MIT press; 2000.

20. Ostrom E. A behavioral approach to the rational choice theory of collective action. The American Political Science Review. 1998; 1-22.

21. Castaldo S. Fiducia e relazioni di mercato: Il mulino; 2002.

22. Castelfranchi C, Falcone R. Trust Theory: A Socio-Cognitive and Computational Model. Primeira ed.: Wiley Publishing; 2010.

23. Bromley DB. Reputation, image, and impression management New York: Wiley; 1993.

24. Marsh S. Formalising Trust as a Computational Concept. Tese Doutorado. University of Stirling, Department of Computing Science; 1994.

25. Sichman S, Demazeau Y. A first attempt to use dependence situatons as a decision criterion for choosing partners in multi-agent systems. In Proceedings of ECAI-94 Workshop on Decision Theory for DAI Applications. 1994.

26. Castelfranchi C, Miceli M, Cesta A. Dependence Relations Among Autonomous Agents. Decentralized AI 3 Proceedings of the Third European Workshop on Modelling Autonomous Agents in a Multi-Agent World (MAAMAW-91). 1992 December; 215-231. 